\title{
Making graphene nanoribbons photoluminescent
}

\author{
B.V. Senkovskiy, ${ }^{*, \dagger}, @$ M. Pfeiffer,,${ }^{\ddagger}$ S.K. Alavi, ${ }^{\ddagger}, \mathbb{l}, @$ A. Bliesener, ${ }^{\dagger}$ J. Zhu, ${ }^{\dagger}$ \\ S. Michel,$^{\dagger}$ A.V. Fedorov $,{ }^{\dagger}, \S^{\prime} \|$ R. German,${ }^{\dagger}$ D. Hertel,${ }^{\ddagger}$ D. Haberer, ${ }^{\perp}$ L. Petaccia, ${ }^{\#}$ \\ F.R. Fischer, ${ }^{\perp}$ K. Meerholz, ${ }^{\ddagger}$ P.H.M. van Loosdrecht, ${ }^{\dagger}$ K. Lindfors, ${ }^{*}, \ddagger$ and
}

A. Grüneis*,†

II. Physikalisches Institut, Universität zu Köln, Zülpicher Strasse 77, 50937 Köln, Germany, Department für Chemie, Universität zu Köln, Luxemburger Strasse 116, 50939 Köln, Germany, Institut für Angewandte Physik der Universität Bonn, Wegeler Strasse 8, 53115 Bonn, Germany, St Petersburg State University, Ulianovskaya 1, St Petersburg 198504, Russia, IFW Dresden, P.O. Box 270116, Dresden D-01171, Germany, Department of Chemistry, University of California at Berkeley, Tan Hall 680, Berkeley, CA 94720, USA, and Elettra Sincrotrone Trieste, Strada Statale $14 \mathrm{~km}$ 163.5, 34149 Trieste, Italy

E-mail: senkovskiy@ph2.uni-koeln.de; klas.lindfors@uni-koeln.de; grueneis@ph2.uni-koeln.de

\begin{abstract}
We demonstrate the alignment-preserving transfer of parallel graphene nanoribbons (GNRs) onto insulating substrates. The photophysics of such samples is characterized by polarized Raman and photoluminescence (PL) spectroscopies. The Raman scattered light and the PL are

\footnotetext{
${ }^{*}$ To whom correspondence should be addressed

${ }^{\dagger}$ II. Physikalisches Institut, Universität zu Köln, Zülpicher Strasse 77, 50937 Köln, Germany

¥Department für Chemie, Universität zu Köln, Luxemburger Strasse 116, 50939 Köln, Germany

IInstitut für Angewandte Physik der Universität Bonn, Wegeler Strasse 8, 53115 Bonn, Germany

${ }^{\S}$ St Petersburg State University, Ulianovskaya 1, St Petersburg 198504, Russia

IFW Dresden, P.O. Box 270116, Dresden D-01171, Germany

${ }^{\perp}$ Department of Chemistry, University of California at Berkeley, Tan Hall 680, Berkeley, CA 94720, USA

\#Elettra Sincrotrone Trieste, Strada Statale 14 km 163.5, 34149 Trieste, Italy

${ }^{\circledR}$ Contributed equally to this work
} 
polarized along the GNR axis. The Raman cross section as a function of excitation energy has distinct excitonic peaks associated with transitions between the one-dimensional parabolic subbands. We find that PL of GNRs is intrinsically low but can be strongly enhanced by blue laser irradiation in ambient conditions or hydrogenation in ultra-high vacuum. These functionalization routes cause the formation of $\mathrm{sp}^{3}$ defects in GNRs. We demonstrate laser writing of luminescent patterns in GNR films for maskless lithography by controlled generation of defects. Our findings set the stage to further exploration of the optical properties of GNRs on insulating substrates and in device geometries.

KEYWORDS: graphene, nanoribbons, hydrogenation, photoluminescence, Raman, defects

One-dimensional (1D) graphene nanoribbons (GNRs) are ideal candidates for materials in the inevitable post-silicon electronics era which will require sub-nanometer structuring. The large versatility in electronic properties makes it possible to use them as an active element in devices ${ }^{1-4}$ with a tunable band gap ${ }^{5-8}$ or for metallic interconnects that could outperform copper contacts with a similar dimension ${ }^{9}$. Atomically precise engineering of GNRs is possible thanks to the bottom-up fabrication approach ${ }^{10-13}$. This technique also allows to produce intermolecular junctions between GNRs of different width ${ }^{14}$ and substitutional doping of GNRs ${ }^{15,16}$. Semiconducting armchair GNRs with $N=7$ carbon atoms width (7-AGNRs) can be grown on vicinal surfaces of $\mathrm{Au}(788)$ and have a long-range parallel alignment over the whole single-crystal substrate ${ }^{17,18}$. For an aligned GNR film, the 1D nature is evident in a strong polarization dependent absorption of light. Optical reflectance anisotropy measurements of 7-AGNRs on $\mathrm{Au}(788)$ have yielded a lowest peak in the absorption at $\mathrm{E}_{11}=2.1 \mathrm{eV}$ which is excitonic in nature ${ }^{19}$. Here $\mathrm{E}_{11}$ is the energy of the optical transition between the highest valence to the lowest conduction band. The energetically higher transitions between the $i$-th valence and conduction band are denoted $\mathrm{E}_{i i}$. The observed anisotropy is common for $1 \mathrm{D}$ materials such as carbon nanotubes ${ }^{20,21}$ and rooted in depolarization effects, that is the suppression of an applied external electromagnetic field perpendicular to the long axis ${ }^{22}$. Moreover, the dipole selection rules for optical transitions depend on the light polarization and state that for GNRs, optical transitions between van-Hove-singularities from the same sub-band 
are allowed only for light polarized along the GNR axis ${ }^{23}$. Previous studies of GNRs on metallic substrates did not report photoluminescence (PL) because the metallic substrates typically quench $\mathrm{PL}^{24}$. PL was observed from GNRs encapsulated inside carbon nanotubes ${ }^{25,26}$. The width of these nanoribbons is determined by the nanotube diameter distribution. Since chirality pure carbon nanotube samples are still challenging, the nanoribbons obtained in this way have non-uniform widths ${ }^{25}$. Moreover such samples are not aligned and cannot be contacted electrically.

From a device perspective, the alignment of GNRs is equally important. For example, in a field effect transistor, one desires to have the GNR orientation along the source-drain direction to have charge transport along the GNR axis and in optoelectronic devices the absorption and emission of light is most efficient for polarization along the GNR axis. Hence, in order to fully exploit the potential of GNRs for nanoelectronics and optoelectronics, an alignment-preserving transfer method onto insulating surfaces is needed. Very recently, the transfer of chevron type GNRs by etching away the $\mathrm{Au}(111) / \mathrm{MICA}$ substrate has been demonstrated ${ }^{27}$. This procedure involves dissolving the Au substrate in acid and is therefore not feasible for a single crystal substrate. Hence, this method does not work for transferring aligned GNRs from the vicinal surfaces of single-crystal substrates.

The present manuscript addresses these issues. An important point regarding PL of GNRs is that theory predicts that the $N=3 p+1$ family (where $p$ is an integer) has an optically forbidden (dark) excitonic state with an energy close to $E_{11}{ }^{28,29}$. Therefore the technologically important 7-AGNRs which have an electron energy band gap in the visible spectral range are not expected to have PL in their intrinsic state. This situation is similar to carbon nanotubes ${ }^{30-32}$, where the generation of defects with at an energy level below the dark exciton results in a stronger PL $^{33}$. Also graphene can be made luminescent using defects ${ }^{34,35}$. The present work establishes the techniques and the physics for obtaining PL brightening in GNRs.

This manuscript is organized as follows. We first describe a procedure how to transfer aligned 7-AGNRs from $\mathrm{Au}(788)$ to insulating substrates ( $\mathrm{Si}$ and quartz wafers). We then characterize the transferred samples using Raman and PL regarding their optical transition energies and their 
alignment. We subsequently induce PL using blue light illumination in ambient conditions and by in-situ hydrogenation in ultra-high vacuum (UHV). In the context of blue light illumination we also show an application how to write PL active defect patterns.

We now turn to the description of the transfer of large area $\left(\mathrm{cm}^{2}\right)$ monolayers of aligned 7AGNRs from $\mathrm{Au}(788)$ onto insulating substrates. Prior to the transfer we characterized samples by a combination of angle-resolved photoemission spectroscopy, scanning-tunneling microscopy, and low energy electron diffraction (see supporting information Figure S1). The Raman measurements of 7-AGNRs on $\mathrm{Au}(788)$ using linearly polarized light yield a highly anisotropic signal, as shown in Figure 1a. We have used the $532 \mathrm{~nm}$ laser line which is close to the $\mathrm{E}_{22}$ transition for 7AGNRs/Au(788) ${ }^{19}$. The most pronounced Raman-active modes are the $G$ mode, corresponding to the carbon-carbon bonds stretching along the ribbon, the $D$ mode which can be related to defects or the edges of $\mathrm{GNRs}^{36}$, the radial breathing like mode $(R B L M)$ related to the ribbon width expansion, and confinement-derived vibration modes ${ }^{37}$ at $1225 \mathrm{~cm}^{-1}$ and $1263 \mathrm{~cm}^{-1}$, respectively. In Figure 1a, $I_{\|}$and $I_{\perp}$ denote the Raman spectra measured for the incident and scattered light polarized along and perpendicular to the step edges of $\mathrm{Au}(788)$ with a ratio $I(G)_{\|} / I(G)_{\perp}=11.4$ (peak value of the $G$ band intensity). The observed anisotropy $I_{\|} / I_{\perp}$ is a measure of how well the alignment is preserved after transfer. Such a quick and non-destructive way to check the alignment is e.g. important for device fabrication from GNRs which requires knowledge of the orientation.

We applied the so-called bubbling transfer method which was developed for CVD grown graphene $^{38,39}$. As we will show, the advantage of this technique is that it retains the GNR parallel alignment over hundreds of microns. Furthermore, it does not affect the single crystalline surface of the substrate ${ }^{40}$. Figure $1 \mathrm{~b}$ depicts the Raman spectra of GNRs after bubbling transfer onto a $\mathrm{Si}$ wafer with $300 \mathrm{~nm} \mathrm{SiO}_{2}$ for polarization parallel and perpendicular to the GNRs. The inset to Figure 1b depicts an optical micrograph of the transferred sample. The GNR coverage is visible as a slightly darker area in the middle of the micrograph. The optical contrast of a GNR monolayer is similar to graphene ${ }^{41,42}$. The darker regions in some areas close to the corners of the micrograph correspond to multiple 7-AGNRs layers due to wrinkles. After transfer we have 

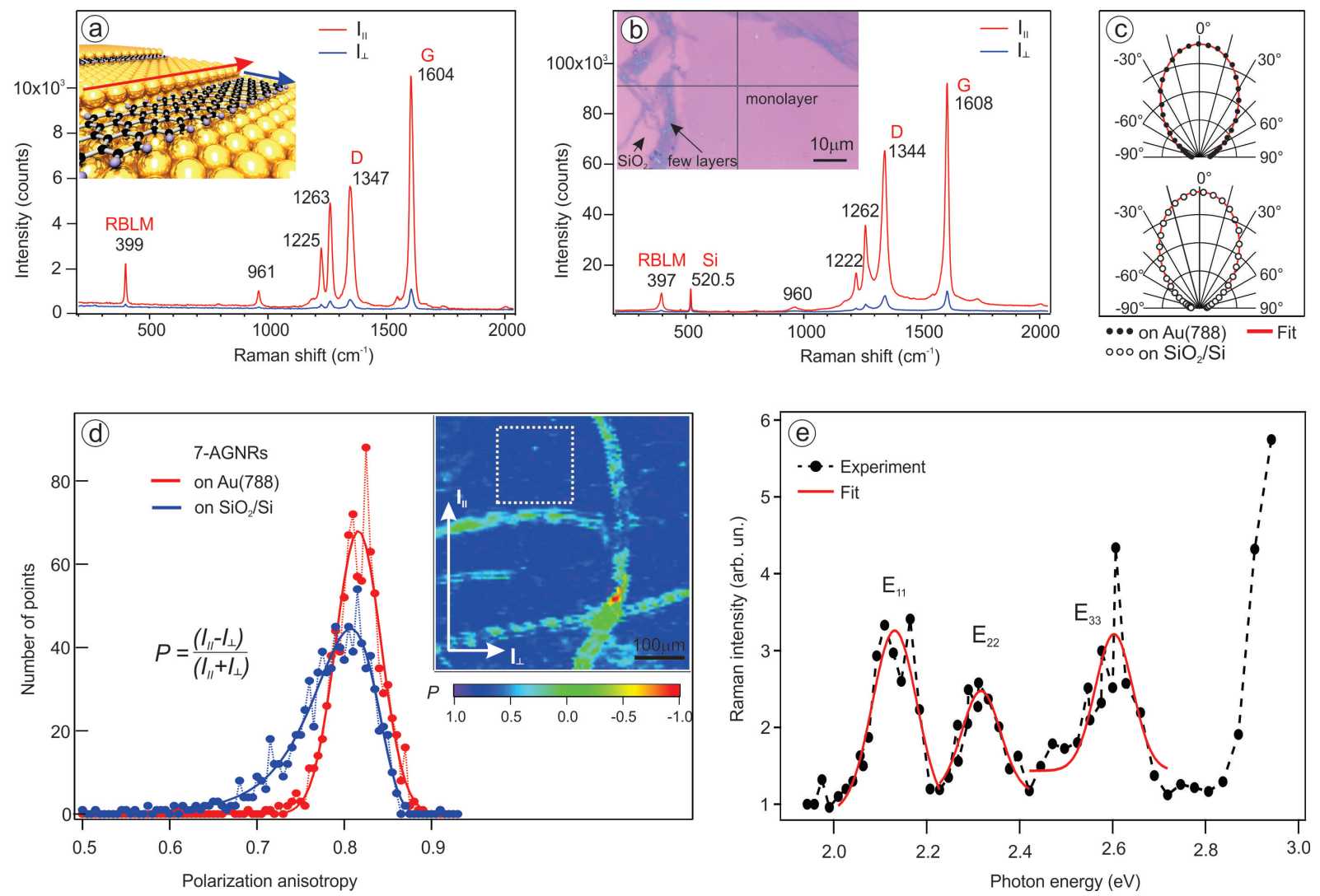

Figure 1: Polarized Raman spectra of 7-AGNRs (a) before and (b) after the transfer from Au(788) onto $\mathrm{SiO}_{2}$, measured inside a UHV Raman system with a $532 \mathrm{~nm}$ wavelength laser with two polarizations: along $\left(I_{\|}\right)$and perpendicular $\left(I_{\perp}\right)$ to the alignment direction. The inset in (a) shows a sketch of aligned 7-AGNRs on $\mathrm{Au}(788)$ and the inset in (b) an optical micrograph of transferred ribbons onto $\mathrm{SiO}_{2}$. (c) Polar diagrams of the Raman intensity of the $G$ mode for 7-AGNRs/Au(788) [top] and on $\mathrm{SiO}_{2}$ [bottom]. The fit of the polar diagram using Eq. 1 is also shown. (d) Histogram plots of Raman polarization anisotropy $P$ for 7-AGNRs on $\mathrm{Au}(788)$ and on $\mathrm{SiO}_{2}$ measured over the $150 \times 150 \mu \mathrm{m}^{2}$ area (points represent the data and the solid lines are guides to the eye). The inset shows the spatial distribution of $P$ for $7-\mathrm{AGNRs} / \mathrm{SiO}_{2}$. The area marked by the dashed line was used for the histogram plot. (e) The Raman excitation profile for 7-AGNRs on quartz as a function of photon energy. The peaks in the Raman intensity at exciton energies $\mathrm{E}_{i i}$ are indicated. Gaussian fits of the individual peaks in the resonance Raman profile are shown. 
$I(G)_{\|} / I(G)_{\perp}=11.1$ for a measurement at a single point. Therefore we conclude that the GNR alignment is preserved in this sample spot. The transfer process causes a small increase in the $D / G$ band intensity ratio which is a measure of disorder ${ }^{43}$ due to remains from the transfer procedure and defects ${ }^{44}$. The $D / G$ ratio is equal to $0.54(0.71)$ before(after) the transfer. Interestingly, the intensity of the Raman spectra for $7-\mathrm{AGNRs} / \mathrm{SiO}_{2}$ is increased by a factor $\sim 9$ relative to the spectrum taken on 7-AGNRs/Au(788). This effect can be ascribed to the change in optical environment because the $\mathrm{SiO}_{2} / \mathrm{Si}$ interface causes multiple reflections and interference effects ${ }^{45,46}$. We also find that the $G$ mode is blue-shifted by $4 \mathrm{~cm}^{-1}$ on $\mathrm{SiO}_{2} / \mathrm{Si}$ relative to the sample on $\mathrm{Au}(788)$. Interestingly, a similar observation (blue-shifts up to $12 \mathrm{~cm}^{-1}$ ) has been made for graphene on $\mathrm{SiO}_{2} / \mathrm{Si}$ compared to metals ${ }^{47}$. For graphene on a substrate with many defects, the blue-shift of the $\mathrm{G}$ mode was explained by the presence of charged impurities ${ }^{47}$. Indeed, charged impurities in the $\mathrm{SiO}_{2}$ substrate are known to be a dominant source of scattering in graphene ${ }^{48,49}$. We believe that the same is true for $7-\mathrm{AGNRs}_{\mathrm{SiO}}$.

Figure 1c shows the polar diagrams of the Raman $G$ mode intensity for 7-AGNRs on Au and on $\mathrm{SiO}_{2}$ as a function of angle between the electric field vector and the alignment direction. From electromagnetic theory for a point-like Hertz dipole and experiments on carbon nanotubes, we expect ${\operatorname{a~} \cos ^{4} \phi \text { law for the Raman intensity }}^{20,21}$. Here $\phi$ is the angle between the laser polarization and the GNR axes. We model the alignment of GNRs by a Gaussian distribution of angles of the GNR axis to the orientation of the $\mathrm{Au}(788)$ terraces. This enables us to extract the average misaligment before and after the transfer from the width of the Gaussian distribution. To that end we fit the polar dependence of the Raman signal $I(\theta)$. Here $\theta$ is the angle with respect to the terraces. The $I(\theta)$ is given by

$$
I(\theta)=\frac{A}{\sigma \sqrt{2 \pi}} \int_{-\pi}^{\pi} \cos ^{4}\left(\phi^{\prime}-\theta\right) \exp \left(-\frac{\left(\phi^{\prime}-\theta\right)^{2}}{2 \sigma^{2}}\right) d \phi^{\prime} .
$$

As a relevant fit result we obtain $\sigma$, the value of the width of the GNR alignment distribution (see Eq. 1). We find equal values of $\sigma=9^{\circ}$ for the GNR alignment distribution before and after the transfer. This indicates that the alignment of the GNRs film is not deteriorating within the area 
of a randomly chosen laser spot $\left(1 \mu \mathrm{m}^{2}\right)$ after the bubbling transfer. The polar diagrams for the other Raman active phonons are shown in the supporting information (Figure S1).

Let us now turn to the investigation of the GNR alignment over larger areas. For a quantitative description of the alignment, we define the Raman polarization anisotropy $P$ for all Raman active modes as $P=\left(I_{\|}-I_{\perp}\right) /\left(I_{\|}+I_{\perp}\right)$. For perfect GNR alignment parallel (perpendicular) to the step edges we have $P=1(P=-1)$ and for a completely random alignment we have $P=0$. Histogram plots of $P$ for a $150 \times 150 \mu \mathrm{m}^{2}$ area of 7-AGNRs before and after the transfer are shown in Figure 1d (this area is not identical with the area of the photograph in Figure 1b). For 7-AGNRs/Au(788) the histogram has a symmetric shape with the center at $P=0.82$. After the transfer the histogram acquires an asymmetric shape with the maximum shifted to $P=0.81$. As a result the distribution peak intensity is reduced by a factor of 1.5 . It is clear that the asymmetry in the distribution of $P$ comes from the fact that the alignment of a fraction of the GNRs deteriorates during the transfer process. The inset of Figure $1 \mathrm{~d}$ shows the spatial distribution of $P$ for $7-\mathrm{AGNRs}_{\mathrm{SiO}}$ with the marked rectangle from which the histogram was computed. It can be seen that large areas have $P \sim 0.8$ which proves the GNR alignment over hundreds of $\mu \mathrm{m}$ distances. There are elongated and bent regions, which feature $P=0$. We ascribe them to multiple folds that can appear during the transfer process. We can conclude that, using the bubbling transfer approach, it is possible to obtain large areas parallel aligned GNRs on an arbitrary substrate.

Having demonstrated the successful transfer of aligned GNRs to insulating substrates we next study the electronic states of the material. For this purpose we measure resonance Raman spectra for different photon energies of the incident light, which is polarized along the GNRs (see methods for details on the experiments). Figure 1e shows the resonance Raman signal of the $G$ band phonon as a function of the photon energy of the incident light (the raw spectra are shown in Figure S2 of the supporting information). The Raman excitation spectrum displays peaks at certain energies, which we associate with the excitons $\mathrm{E}_{i i}$ in the GNRs ${ }^{50}$. We determine the positions as $\mathrm{E}_{11}=$ $2.1 \mathrm{eV}, \mathrm{E}_{22}=2.3 \mathrm{eV}$, and $\mathrm{E}_{33}=2.6 \mathrm{eV}$. The rise of the Raman cross section at energies higher than $\mathrm{E}_{33}$ is ascribed to a combination of absorption of light at higher $\mathrm{E}_{i i}$ transitions and at the saddle 
point singularity which appears in all $\mathrm{sp}^{2}$ carbon materials. For graphene, this transition occurs at $4.62 \mathrm{eV}^{51}$.

Let us now turn to the photoluminescence of GNRs. When GNRs are illuminated with focused $440 \mathrm{~nm}$ wavelength light in a laser scanning confocal microscope under ambient conditions we observe weak emission without distinct spectral features as illustrated by the PL spectrum shown in Figure 2a (see methods for details on the PL experiments). For a defect-free sample, the PL is expected to be dark. Surprisingly, when the sample is continuously exposed to $440 \mathrm{~nm}$ radiation the emission brightens after one minute and a peak at approximately $1.8 \mathrm{eV}$ emerges in the spectrum. Regarding the kinetics of defect generation, we find that for an incident intensity at the microscope focus of approximately $100 \mathrm{~kW} / \mathrm{cm}^{2}$, it takes approximately five minutes for the brightening to saturate depending on the wavelength. We attribute the increased emission to the generation of defects in GNRs via a photochemical reaction. The initially very weak fluorescence before exposure to $440 \mathrm{~nm}$ light is a signature of the existence of dark excitons energetically either degenerate with the lowest lying optically active exciton $\mathrm{E}_{11}$ or positioned below this bright transition as predicted in theoretical studies $28,29,52,53$. The weak but not completely absent PL emission before exposure to blue light is ascribed to the presence of defects, which were generated either during sample growth or transfer, or which formed in laboratory conditions after the sample was removed from UHV. Sample transfer as the cause of the defects is supported by the fact that we observed an increase in the $D / G$ Raman ratio (see Figure 1) although we remark that not all defects are expected to result in brightening of luminescence.

It is important to distinguish between the wavelengths of the light needed to efficiently generate defects and the light used to excite PL. We find that for defect generation, the sample must be exposed to light with wavelengths shorter than approximately $510 \mathrm{~nm}$. For longer wavelengths the PL remains dim independent of the photon energy used to excite PL. Once we have generated defects, we can probe the dependence of the PL spectrum on the exciting light wavelength. To that end we have performed PL measurements (data not shown here) varying the excitation wavelength from $420 \mathrm{~nm}$ to $590 \mathrm{~nm}$ and observe a similar emission spectrum for all excitation wavelengths. This 
demonstrates that the observed emission originates from an energetically well-defined transition.

Figure $2 \mathrm{~b}$ shows that the PL displays a significant polarization anisotropy across the spectral range of the emission. The emission is maximum for polarization along the ribbons. The fact that the emission is polarized definitively proves that the emission originates from the ribbons. Figure S5 in the supporting information depicts a quantitative characterization of the degree of polarization. The linearly polarized PL emission is typical for $1 \mathrm{D}$ objects. The observed brightening of the PL is similar to that observed in carbon nanotubes ${ }^{33,54,55}$. For unperturbed nanotubes fluorescence is very weak due to dark excitons at the energetic position of the optically active transition. Photochemically inducing defects into the nanotubes modifies the energy level structure resulting in a significant increase in fluorescence. In the present case, the photochemical reaction between 7-AGNRs and oxygen and moisture of ambient air, but also a photoinduced reaction of 7-AGNRs with the $\mathrm{SiO}_{2}$ surface ${ }^{56}$ are thought to be key for the generation of defects that lead to PL. This is suggested by the observation that the rate of the photoinduced modification is suppressed if blue laser illumination is performed under high-vacuum conditions (data not shown here). The reaction of oxygen with graphene can form more than one type of $\mathrm{sp}^{3}$ defect in the basal plane (e.g. ether, epoxide, ketone) but also edge defects ${ }^{35,57,58}$. This is also known from graphene oxide which is formed by oxidation of graphene using strong acids in ambient conditions ${ }^{35}$. The PL of graphene oxide occurs at a very similar energy (maximum PL at 1.8 to $1.9 \mathrm{eV}$ ) 34,35 than observed here for GNRs.

We have compared the Raman spectrum before and after inducing defects photochemically. We observe no change in the position of the $D$ and $G$ Raman modes but a $25 \%$ decrease in the intensities of both lines (see supporting information Figure S3b). The decrease eventually saturates in similar fashion as the PL signal increases. This further demonstrates that the photochemical modification does not result in major degradation of the GNR structure. Regarding the time constants we note that PL decay is equal to the instrument response function and faster than 500 ps. This points to a fluorescence process instead of slow phosphorescence and towards a significant nonradiative rate, possibly due to the dark exciton at $\mathrm{E}_{11}$ (see supporting information Figure S4a). 
Another important point is whether PL bleaches after certain durations of excitation. To that end we collected PL over longer time frames and can confirm the absence of bleaching (see supporting information Figure S4b).

Due to the ambient conditions of the experiment, a direct proof of the $\mathrm{sp}^{3}$ nature of the defect by X-ray photoemission (XPS) and near edge X-ray absorption (NEXAFS) spectroscopies is not possible as this would require in-situ functionalization in UHV conditions. At the end of the description of the results we present in-situ studies of hydrogenated 7-AGNRs which allow for a combined PL, XPS and NEXAFS investigation of the chemical nature and luminescent properties of hydrogen defects.

We next demonstrate an application of the laser-induced PL brightening. By scanning the laser focus we can write luminescent patterns in the GNR film. Figure 2c shows spectrally integrated fluorescence micrographs of a region of the sample where we have exposed the pattern in the form of the letters "UoC" using $440 \mathrm{~nm}$ wavelength light. The emission is significantly brighter in the regions exposed to the blue light. However, the surrounding region is not completely dark and demonstrates that a small number of defects are present as discussed in the preceeding section. The emission process is polarization dependent both in the excitation and emission as a consequence of the high level of alignment of the ribbons. The four panels show the fluorescence under the four possible combinations of excitation and emission light polarized parallel and perpendicular to the GNR axis. The two arrows in each panel denote the light polarization for excitation (left arrow) and emission (right arrow). Here, a vertical arrow denotes light polarization along the GNRs and a horizontal arrow denotes light polarization perpendicular to the GNRs. It can be seen that the brightest PL pattern appears for both, excitation and emission light polarized along the GNR [upper left panel of Figure 2c]. If either excitation or emission are polarized perpendicular to the GNR, we obtain a medium intensity PL pattern. This situation corresponds to the upperright and lower-left panels of Figure 2c. Finally, the weakest PL pattern is observed, if both, the polarization of excitation and emission are perpendicular to the GNR axis. This situation is depicted in the bottom-right panel of Figure 2c. Both, the bright, photochemically modified regions 

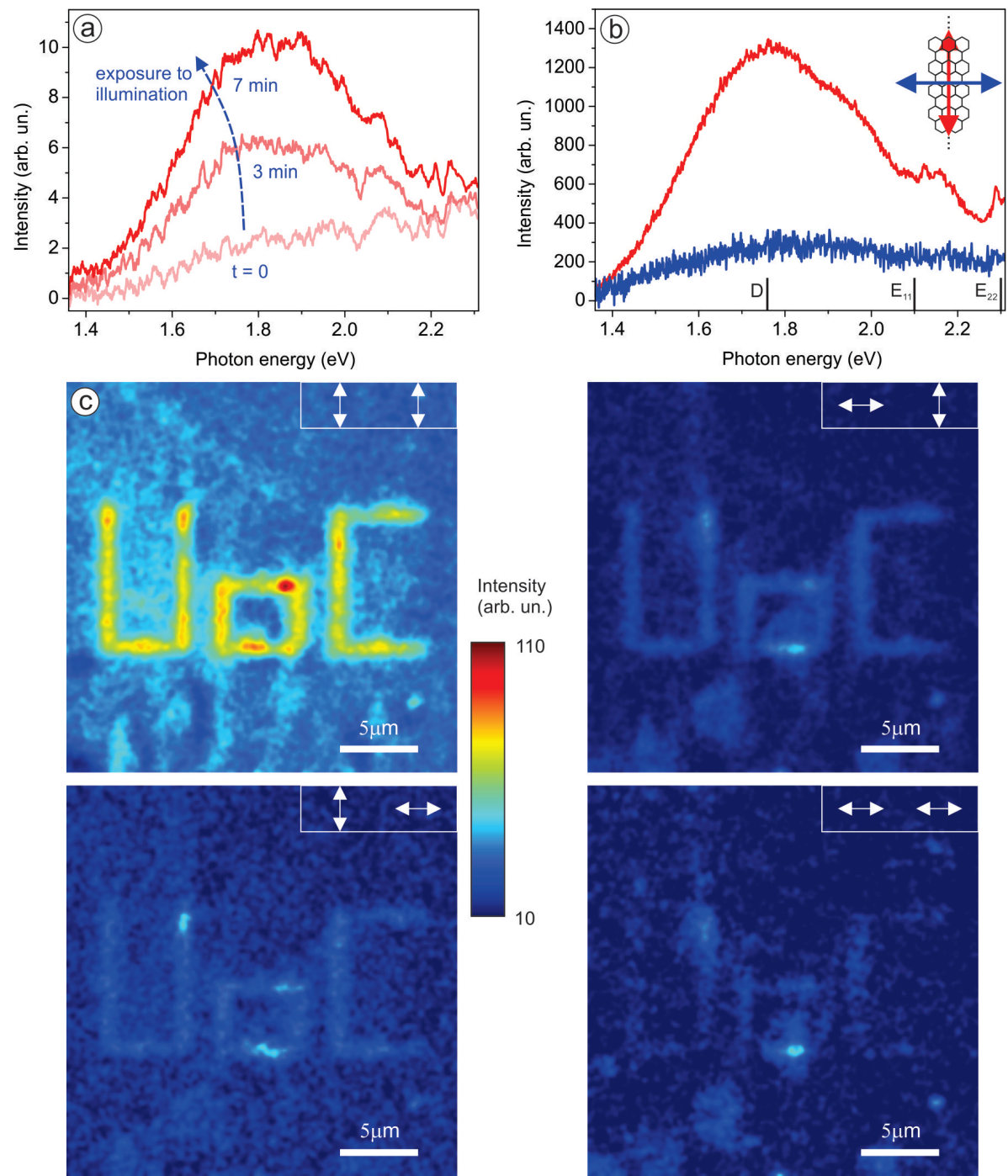

Figure 2: (a) The PL of 7-AGNRs on quartz (peak at $\sim 1.8 \mathrm{eV}$ ) brightens when the sample is exposed to blue light. (b) The PL is partially polarized along the GNR axis demonstrating its origin in the GNR. Here the spectra for polarizer and analyzer both parallel to the ribbon axis (red solid line) and both perpendicular to the ribbon axis (blue solid line) are shown. The emission peaks are at significantly lower photon energy (marked with $D$ ) than the excitons $\mathrm{E}_{11}$ and $\mathrm{E}_{22}$. (c) Scanning a $440 \mathrm{~nm}$ laser focus allows the writing of luminescent patterns on GNR monolayers. Here the letters "UoC" have been rendered bright. The four panels correspond to the four possible orientations of polarizer and analyzer with respect to the GNR axis and are indicated by arrows. The first (second) arrow denotes the polarization of the exciting (analyzed) light. The vertical direction corresponds to light polarization along the GNR axis according to the sketch of a GNR in the inset to (b). 
(the letters "UoC") and the surrounding areas show the same polarization dependence over the spectral range covering the PL emission. This indicates that light exposure does not destroy the ribbons (see supporting information Figure S5). Thus the writing of luminescent patterns could be useful in situations where small structures are needed. Maskless lithography is an important tool in nanotechnology and has previously been performed, e.g., by aqueous electro-oxidation of diamond-like carbon films using an atomic force microscope ${ }^{59}$. The present approach does not require a local probe and is therefore faster and easier to perform.

\section{Hydrogen induced photoluminescence}

Blue laser illumination in ambient conditions is a viable route to write bright photoluminescent patterns. However, controlled experiments in an UHV environment are needed to correlate the atomic structure of the defect to the appearance of PL. In order to investigate the PL from a defined defect (characterized by XPS), other routes for defect generation are needed. Here we show how to induce $\mathrm{sp}^{3}$ defects in 7-AGNR samples by hydrogenation in UHV conditions.

Hydrogen $\mathrm{sp}^{3}$ defects in the basal plane of many graphene based carbon materials can be readily induced by exposure to a beam of atomic hydrogen which form $\mathrm{C}-\mathrm{H}$ bonds perpendicular to the graphene sheet ${ }^{60-64}$. The hydrogenation of transferred GNRs on $\mathrm{SiO}_{2} / \mathrm{Si}$ and the PL experiments were performed inside a homebuilt UHV PL system (see methods) without exposing the sample to air after hydrogenation. In such a well-defined experiment, the PL can be definitely ascribed to $\mathrm{sp}^{3}$ defects formed by $\mathrm{C}-\mathrm{H}$ bonds. The $\mathrm{sp}^{3}$ character of these defects can be confirmed by XPS and NEXAFS spectroscopies (see supporting information, Figure S6). Figure 3a depicts the PL spectra of the sample inside UHV before hydrogenation measured with a $532 \mathrm{~nm}$ laser with light

polarized parallel and perpendicular to the GNRs. The spectra exhibit strong Raman modes and their higher orders. It is seen that intrinsically PL from 7-AGNRs is very weak. Exposure to a beam of atomic hydrogen ${ }^{63,64}$ induces defects where a carbon atom forms three C-C bonds and one $\mathrm{C}-\mathrm{H}$ bond, as illustrated in Figure $3 \mathrm{~b}$. It can be seen from the Figure $3 \mathrm{c}$ that the hydrogenation (10 minutes exposure) leads to a strong polarized PL peak with a maximum at around $1.8 \mathrm{eV}$. 
At the same time, the Raman signal from ribbons becomes weaker. We believe that this is due to the fact that the number of resonant $\mathrm{sp}^{2}$ bonds is reduced. We also observe disorder induced broadening of the Raman lines. This is presumably due to the random nature of chemisorbed $\mathrm{H}$ atoms. Longer exposure (40 minutes) to atomic hydrogen corresponds to the maximum amount of hydrogen that can be chemisorbed to 7-AGNRs. A quantitative analysis of the PL of hydrogenated GNRs yields an increase by a factor of 5 for 10 min hydrogenation (see supporting information Figure S7 and Table S1). Note, the PL intensity (as well as the Raman intensity) can be affected by interference effects and thus the obtained values for the PL intensity enhancement depend on the $\mathrm{SiO}_{2}$ thickness. XPS allows us to estimate the amount of hydrogenated $\mathrm{C}$ atoms for the 40 minutes exposure. We obtain a maximum value of $\sim 25 \%$ (see supporting information Figure S6). This is identical to the maximum hydrogen coverage observed for epitaxial single-layer graphene ${ }^{64}$. The PL spectrum in Figure 3d displays further reduction of the Raman peaks and an increase of the PL intensity relative to the Raman signal. However, the absolute intensity of PL is decreased when compared to the 10 minutes exposure. This is due to the fact that the PL intensity depends upon the absorption of incident light. We speculate that this absorption is becoming less effective as more and more $\mathrm{sp}^{2}$ bonds are broken. Thus, to observe the maximum PL signal from 7-AGNRs, there is a compromise between the luminescence and absorption efficiency

Finally we discuss the similarity of the PL spectra of defective 7-AGNRs using the two routes for engineering defects (blue laser illumination and hydrogenation). The fact that the peak positions of the PL for both kinds of defects are very similar to each other is not surprising because the electronic structure of these $\mathrm{sp}^{3}$ defects is similar. This is supported by a work on PL from defective nanotubes using nine different functional groups that bond in an $\mathrm{sp}^{3}$ manner ${ }^{54}$. The emission energies of these nine $\mathrm{sp}^{3}$ defects were within an energy window of $40 \mathrm{meV}$. We also wish to point out that a more detail comparison of the PL of different defects would require a dedicated study using identical excitation energies and substrates. In particular, it is well known that the thin $\mathrm{SiO}_{2}$ layer on top of the $\mathrm{Si}$ wafer acts as a cavity and can enhance/supress the emission of certain wavelengths. 

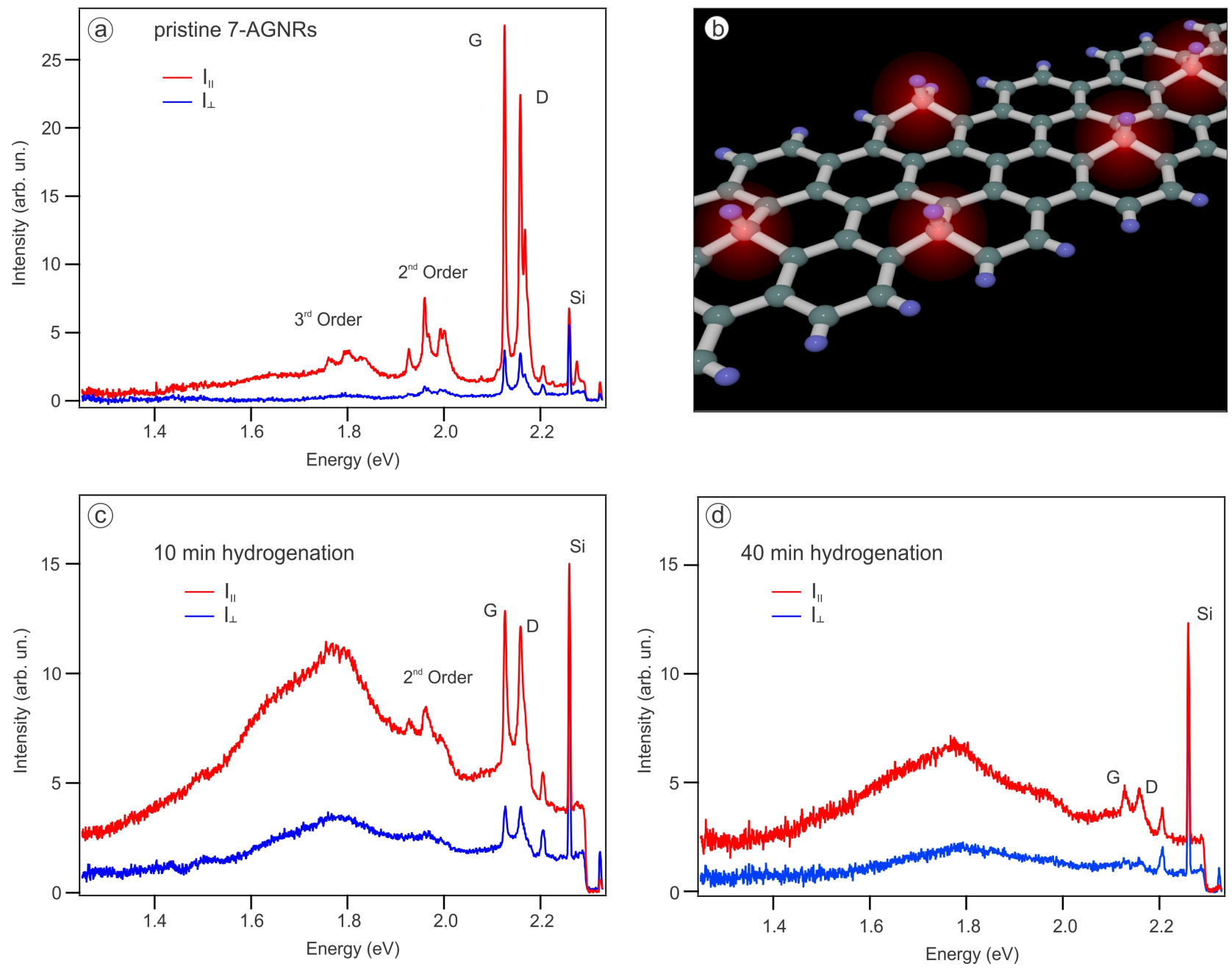

Figure 3: (a) PL and Raman spectra of transferred 7-AGNRs/SiO ${ }_{2}$ measured inside a UHV chamber after annealing at $300^{\circ} \mathrm{C}$. Red and blue lines indicate spectrum recorded along $\left(I_{\|}\right)$and perpendicular $\left(I_{\perp}\right)$ to the alignment direction, respectively. (b) Sketch of the hydrogenated ribbons. (c, d) PL and Raman spectra of the system after 10 minutes and after 40 minutes of atomic hydrogen exposure. A polarized PL peak is induced by $\mathrm{sp}^{3}$ hydrogen defects. 
In conclusion, we have performed a full photophysical characterization using polarized Raman and luminescence measurements of 7-AGNRs on insulating substrates. To that end we have adapted the bubbling transfer method to transfer monolayers of 7-AGNRs from $\mathrm{Au}(788)$ keeping their parallel alignment. We have demonstrated that polarized Raman spectroscopy is a quick and simple tool to analyze the GNR alignment before and after the transfer. The Raman excitation profile as a function of exciting laser energy shows a series of distict peaks which we identified with the $\mathrm{E}_{11}, \mathrm{E}_{22}$ and $\mathrm{E}_{33}$ excitons. Optical excitation with an energy equal to one of the $E_{i i}$ energies enhances the Raman or PL signal.

7-AGNRs in the pristine state are only weakly photoluminescent. This is evidence in favor of dark excitonic states arising from the optically forbidden $E_{12}$ and $E_{21} \operatorname{transition}^{23}$. These dark excitons are expected to be in the vicinity of the lowest bright state in $N=3 p+1$ AGNRs and are the channels for nonradiative relaxation ${ }^{28,29}$. We have demonstrated two ways of inducing photoluminescence with light emission at about $1.8 \mathrm{eV}$ polarized along the GNR axis. The first is by blue laser illumination in ambient conditions and the second by UHV hydrogenation. Laser writing of photoluminescent patterns in GNR monolayers has been demonstrated and could find technological applications as a cheap and quick maskless lithographic method for structuring GNR films.

Regarding future research avenues, it would be interesting to transfer and spectroscopically characterize other GNR chiralities using PL. This is especially true for the GNRs belonging to the $N=3 p$ family for which theory predicts the absence of dark excitons ${ }^{28,29}$ such as the $\mathrm{N}=9$ GNR which has only recently been synthesized and characterized ${ }^{65}$. Moreover, the energy and the intensity of the PL for different defects such as vacancies and $\mathrm{sp}^{3}$ carbon impurities (diamond-like defects) could be investigated. These defects may be formed by ion bombardement or the evaporation of $\mathrm{C}$ atoms onto a GNR sample. Recently it was shown, that atomically precise doping by heteroatoms such as boron can be performed in GNRs ${ }^{15}$. We believe that the investigation of PL in boron doped bottom-up fabricated GNRs could be interesting as the boron atoms modifiy the electronic structure and therefore may affect the PL. The boron site in these ribbons is also 
believed to be chemically active and might act as a chemical anchor for adsorption of molecules or atoms at the boron site. This could be used as a template for atomically precise functionalization by luminescent molecules. Another interesting research direction would be to build optically active heterostructures out of GNRs of different chirality and orientation or between GNRs and 2D materials. In such devices, one could observe exciton transfer between the layers.

\section{Methods}

\section{Growth}

7-AGNRs were synthesized by surface polymerization of 10,10-dibromo-9,9-bianthryl (DBBA) molecules ${ }^{11}$ on $\mathrm{Au}(111)$ and $\mathrm{Au}(788)$ surfaces. The gold substrates were cleaned by three cycles of $\mathrm{Ar}^{+}$sputtering $(800 \mathrm{~V})$ and subsequent annealing at $500{ }^{\circ} \mathrm{C}$. DBBA molecules were evaporated from a quartz crucible using a homebuilt evaporator with a thermocouple attached to the molecule

reservoir. The deposition rate was controlled using a quartz microbalance. About $8 \AA$ of precursor molecules (using the graphite density and Z-factor) were evaporated onto the Au surface which was kept at room-temperature. Hereafter, a two-step polymerization reaction was performed: a $200^{\circ} \mathrm{C}$ annealing step followed by a $400^{\circ} \mathrm{C}$ annealing step, which induce debromization and cyclodehydrogenation reactions, respectively. The annealing was carried out using a computer controlled ramp that increased temperature over several hours.

Characterization STM STM measurements were performed using an Omicron LT-STM with the samples held at $4.5 \mathrm{~K}$ in UHV. A tungsten tip was used for topography and spectroscopic measurements. Topographic images were acquired in constant current mode. $\mathrm{dI} / \mathrm{dV}$ spectra were obtained using the lock-in technique where the tip bias was modulated by a $457 \mathrm{~Hz}, 10 \mathrm{mV}$ (rms) sinusoidal voltage under open-feedback conditions. All STM images were processed with WSxM ${ }^{66}$. XPS, NEXAFS and ARPES XPS and NEXAFS experiments were performed at the German-Russian beamline (RGBL) of the HZB BESSY II synchrotron radiation facility (Berlin, Germany). XPS spectra were measured with photon energy of $490 \mathrm{eV}$ and pass energy of $10 \mathrm{eV}$ in the normal emission geometry. NEXAFS data were obtained in total electron yield mode with an energy resolution of $50 \mathrm{meV}$ close to the $\mathrm{C} \mathrm{K}$-edge. ARPES was carried out at the BaDElPh beamline of 
the Elettra synchrotron radiation facility (Trieste, Italy) using a SPECS Phoibos 150 hemispherical electron analyser. The ARPES spectra were measured using a photon energy of $27 \mathrm{eV}$ in the $2^{\text {nd }}$ $\mathrm{BZ}$ of the GNRs and shifted back by application of the reciprocal lattice vector to the $1^{\text {st }} \mathrm{BZ}$. The samples for XPS, NEXAFS and ARPES measurements were synthesized and checked in the UHV Raman chamber and then transferred under UHV conditions into a special container which was filled then to a slight overpressure (1.1 bar) by high purity Ar gas. Samples in UHV tight vacuum suitcases filled with Argon gas were transported to the load-lock chamber of the ARPES beamline endstation and later gently annealed (at $200^{\circ} \mathrm{C}$ ) under UHV conditions. This method allowed us to achieve reproducible experimental results.

Raman The polarization dependent scanning Raman was performed in back-scattering geometry using a commercial Raman system (Renishaw) integrated in a homebuilt optical UHV chamber where the exciting and Raman scattered light were coupled into the vacuum using a long-working distance microscope objective with an NA of $\sim 0.4$. The laser power on the sample was kept below $1 \mathrm{~mW}$. Raman spectra were calibrated using Si peak at $520.5 \mathrm{~cm}^{-1}$ as well as oxygen peak at $1555 \mathrm{~cm}^{-1}$. A half-wave plate was used to rotate the polarization of the laser beam. To collect a certain polarization of the Raman light, a polarizer and a half-wave plate were inserted before the detector. All Raman spectra and the map in Figure 1 have been measured inside a UHV chamber. The Raman spectra have been normalized to the laser power, integration time and system response. Raman spectra as a function of the energy of the incident photons were acquired using an in-house developed laser-scanning confocal microscope. A supercontinuum laser (NKT SuperK EXTREME) and a variable bandpass filter (NKT SuperK VARIA) provides bright tunable radiation. To narrow the spectral bandwidth of the excitation light we additionally use a tunable grating filter. A second similar tunable filter was used in the detection to remove the incident laser light. The incident light is focussed on the sample using a microscope objective with numerical aperture 0.8 (Olympus MPLFLN). Finally, the spectra were acquired with a grating spectrometer (Princeton Instruments IsoPlane) equipped with a deep-cooled back-illuminated CCD. The strength of the Raman signals were extracted from Lorentzian fits to the Raman G-mode. The spectra were cor- 
rected using the wavelength-dependent sensitivity of the setup. The power in the experiments was kept below $200 \mu \mathrm{W}$ for a diffraction limited spot. Photoluminescence Photoluminescence was measured in a sample-scanning laser scanning confocal microscope. The polarization sensitive measurements were performed with an achromatic half-wave plate and a polarizer in the excitation path. In the detection path we used an optical long-pass filter. We further place a polarizer to act as an analyzer behind the long-pass filter. This is followed by an achromatic quarter-wave plate oriented at $45^{\circ}$ with respect to the analyzer polarization axis. This results in polarization-independent propagation of the luminescence light through the optical train to the detector. All spectra were normalized with the power of the incident light and corrected for the polarization-dependencies of the optical train before the analyzer. The power in the experiments was kept below $150 \mu \mathrm{W}$ for a diffraction limited spot. The PL of hydrogenated 7-AGNRs was measured in-situ using the UHV Raman setup (see above). For PL measurements, a filter with a larger band width than in the Raman experiments has been used.

Hydrogenation of graphene nanoribbons To clean the surface prior hydrogenation the aligned 7-AGNRs $/ \mathrm{SiO}_{2}$ were annealed in the UHV chamber (base pressure was $2 \times 10^{-10} \mathrm{mbar}$ ) at $300^{\circ} \mathrm{C}$ for 12 hours. Hydrogenation of GNRs was performed by cracking $\mathrm{H}_{2}$ molecules in a tungsten capillary which was heated to temperatures above 1800-2000 K. The hydrogen flux was controlled by a needle valve. A pressure of $10^{-6}$ mbar was applied during hydrogenation. Bubbling transfer To transfer 7-AGNRs from $\mathrm{Au}(788)$ onto insulating substrates (300 $\mathrm{nm} \mathrm{SiO}_{2} / \mathrm{Si}$ or quartz) we used the bubbling transfer technique based on the electrochemical delamination process ${ }^{38,39}$. The 7-AGNRs/Au(788) sample was coated with polymethyl methacrylate (PMMA) with a molecular weight of $180 \mathrm{kDa}$ and placed in an electrochemical cell with an $\mathrm{NaOH}$ aqueous solution (1 mol/L). A reference mark on the $\mathrm{Au}(788)$ crystal shows the direction of (111) terraces. At the negatively charged cathode the PMMA/7-AGNRs layer was detached from the Au(788) substrate by the emerging $\mathrm{H}_{2}$ gas bubbles due to the reduction of water. The floating PMMA/7-AGNRs layer was then cleaned in distilled water and placed onto the substrate. To remove PMMA and clean the sample surface we employed acetic acid $\left(96 \%\right.$ in $\left.\mathrm{H}_{2} \mathrm{O}\right)$ and acetone. This treatment removes 
residual PMMA contaminants efficiently ${ }^{67}$.

\section{Acknowledgements}

B.S., S.M., A.F. and A.G. acknowledge the ERC grant no. 648589 'SUPER-2D', funding from DFG projects CRC 1238 (project A1) and GR 3708/2-1. Research supported by the U.S. Department of Energy (DOE), Office of Science, Basic Energy Sciences (BES), under Award no. DESC0010409 (design, synthesis and characterization of molecular building blocks) and the Center for Energy Efficient Electronics Science NSF Award 0939514 (SPM imaging and spectroscopy). The research leading to these results has received funding from the European Community's Seventh Framework Programme (FP7/2007-2013) under grant agreement n.312284 (CALIPSO). We thank Elettra for the allocation of synchrotron radiation beamtimes. The stay at the Elettra synchrotron has been supported by the CERIC-ERIC consortium. The authors thank HZB BESSY II and the Russian-German Laboratory (RGBL) for the beamtime allocation. S.K.A. thanks the Bonn-Cologne Graduate School for Physics and Astronomy and DAAD for financial support. This work has been supported by the University of Cologne through the Institutional Strategy of the University of Cologne within the German Excellence Initiative.

Supporting information STM, ARPES and LEED measurements of the aligned 7-AGNRs on $\mathrm{Au}(788)$. Polar diagram of the Raman intensities of the RBLM and D modes, respectively. Sketch of the bubbling transfer setup, photograph of the transferred 7-AGNR film. Raw energy dependent Raman data. Raman and photoluminescence spectra before and after defect generation. Time resolved fluorescence and instrument response function. Time-dependence of the photoluminescence. Polarization anisotropy for the excitation and emission. Polarization anisotropy of the emission for pristine and illumination defected areas. XPS and NEXAFS of hydrogenated 7-AGNRs on $\mathrm{Au}(111)$. Quantification of photoluminescence increase after hydrogenation.

\section{References}

(1) Chen, Z.; Lin, Y.-M.; Rooks, M. J.; Avouris, P. Physica E: Low-dimensional Systems and Nanostructures 2007, 40, 228 - 232, International Symposium on Nanometer-Scale Quantum 
Physics.

(2) Wang, X.; Ouyang, Y.; Li, X.; Wang, H.; Guo, J.; Dai, H. Phys. Rev. Lett. 2008, 100, 206803.

(3) Kim, W. Y.; Kim, K. S. Nature Nanotechnology 2013, 3, 408-412.

(4) Bennett, P. B.; Pedramrazi, Z.; Madani, A.; Chen, Y.-C.; de Oteyza, D. G.; Chen, C.; Fischer, F. R.; Crommie, M. F.; Bokor, J. Applied Physics Letters 2013, 103, 253114.

(5) Nakada, K.; Fujita, M.; Dresselhaus, G.; Dresselhaus, M. S. Phys. Rev. B 1996, 54, 1795417961.

(6) Yang, L.; Park, C.-H.; Son, Y.-W.; Cohen, M. L.; Louie, S. G. Phys. Rev. Lett. 2007, 99, 186801.

(7) Barone, V.; Hod, O.; Scuseria, G. E. Nano Letters 2006, 6, 2748-2754, PMID: 17163699.

(8) Chen, Y.-C.; de Oteyza, D. G.; Pedramrazi, Z.; Chen, C.; Fischer, F. R.; Crommie, M. F. ACS Nano 2013, 7, 6123-6128.

(9) Naeemi, A.; Meindl, J. D. IEEE Electron Device Letters 2007, 28, 428-431.

(10) Grill, L.; Dyer, M.; Lafferentz, L.; Persson, M.; Peters, M. V.; Hecht, S. Nature Nanotechnology 2007, 2, 687-691.

(11) Cai, J.; Ruffieux, P.; Jaafar, R.; Bieri, M.; Braun, T.; Blankenburg, S.; Muoth, M.; Seitsonen, A. P.; Saleh, M.; Feng, X.; Mullen, K.; Fasel, R. Nature 2010, 466, 470-473.

(12) Ruffieux, P.; Wang, S.; Yang, B.; Sànchez-Sànchez, C.; Liu, J.; Dienel, T.; Talirz, L.; Shinde, P.; Pignedoli, C. A.; Passerone, D.; Dumslaff, T.; Feng, X.; Müllen, K.; Fasel, R. Nature 2016, 531, 489.

(13) Talirz, L.; Ruffieux, P.; Fasel, R. Advanced Materials 2016, 28, 6222-6231. 
(14) Chen, Y.-C.; Cao, T.; Chen, C.; Pedramrazi, Z.; Haberer, D.; de Oteyza, D.; Fischer, F.; Louie, S.; Crommie, M. Nature Nanotechnology 2015, 10, 156.

(15) Cloke, R. R.; Marangoni, T.; Nguyen, G. D.; Joshi, T.; Rizzo, D. J.; Bronner, C.; Cao, T.; Louie, S. G.; Crommie, M. F.; Fischer, F. R. Journal of the American Chemical Society 2015, 137, 8872-8875, PMID: 26153349.

(16) Kawai, S.; Saito, S.; Osumi, S.; Yamaguchi, S.; Foster, A. S.; Spijker, P.; Meyer, E. Nat Commun 2015, 6, 8098.

(17) Linden, S.; Zhong, D.; Timmer, A.; Aghdassi, N.; Franke, J. H.; Zhang, H.; Feng, X.; Müllen, K.; Fuchs, H.; Chi, L.; Zacharias, H. Phys. Rev. Lett. 2012, 108, 216801.

(18) Ruffieux, P.; Cai, J.; Plumb, N. C.; Patthey, L.; Prezzi, D.; Ferretti, A.; Molinari, E.; Feng, X.; Müllen, K.; Pignedoli, C. A.; Fasel, R. ACS Nano 2012, 6, 6930-6935, PMID: 22853456.

(19) Denk, R.; Hohage, M.; Zeppenfeld, P.; Cai, J.; Pignedoli, C. A.; Söde, H.; Fasel, R.; Feng, X.; Müllen, K.; Wang, S.; Prezzi, D.; Ferretti, A.; Ruini, A.; Molinari, E.; Ruffieux, P. Nat. Commun. 2014, 5, 4253.

(20) Duesberg, G. S.; Loa, I.; Burghard, M.; Syassen, K.; Roth, S. Phys. Rev. Lett. 2000, 85, $5436-5439$.

(21) Ichida, M.; Mizuno, S.; Kataura, H.; Achiba, Y.; Nakamura, A. Applied Physics A 2004, 78, $1117-1120$.

(22) Ajiki, H.; Ando, T. Physica B: Condensed Matter 1994, 201, 349 - 352.

(23) Sasaki, K.; Kato, K.; Tokura, Y.; Oguri, K.; Sogawa, T. Phys. Rev. B 2011, 84, 085458.

(24) Johansson, P.; Xu, H.; Käll, M. Physical Review B 2005, 72, 035427. 
(25) Chernov, A. I.; Fedotov, P. V.; Talyzin, A. V.; Suarez Lopez, I.; Anoshkin, I. V.; Nasibulin, A. G.; Kauppinen, E. I.; Obraztsova, E. D. ACS Nano 2013, 7, 6346-6353, PMID: 23795665 .

(26) Lim, H. E.; Miyata, Y.; Fujihara, M.; Okada, S.; Liu, Z.; Arifin,; Sato, K.; Omachi, H.; Kitaura, R.; Irle, S.; Suenaga, K.; Shinohara, H. ACS Nano 2015, 9, 5034-5040, PMID: 25868574.

(27) Chen, Z. et al. Journal of the American Chemical Society 2016, 138, 15488-15496, PMID: 27933922.

(28) Li, Y.; Marvin, L. C.; Steven, G. L. Nano Letters 2007, 7, 3112-3115, PMID: 17824720.

(29) Prezzi, D.; Varsano, D.; Ruini, A.; Marini, A.; Molinari, E. Phys. Rev. B 2008, 77, 041404.

(30) Wang, F.; Dukovic, G.; Brus, L. E.; Heinz, T. F. Science 2005, 308, 838-841.

(31) Maultzsch, J.; Pomraenke, R.; Reich, S.; Chang, E.; Prezzi, D.; Ruini, A.; Molinari, E.; Strano, M. S.; Thomsen, C.; Lienau, C. Phys. Rev. B 2005, 72, 241402.

(32) Srivastava, A.; Htoon, H.; Klimov, V. I.; Kono, J. Phys. Rev. Lett. 2008, 101, 087402.

(33) Miyauchi, Y.; Iwamura, M.; Mouri, S.; Kawazoe, T.; Ohtsu, M.; Matsuda, K. Nat Photon 2013, 7, 715-719.

(34) Gokus, T.; Nair, R. R.; Bonetti, A.; Böhmler, M.; Lombardo, A.; Novoselov, K. S.; Geim, A. K.; Ferrari, A. C.; Hartschuh, A. ACS Nano 2009, 3, 3963-3968.

(35) Loh, K. P.; Bao, Q.; Eda, G.; Chhowalla, M. Nat Chem 2010, 2, 1015-1024.

(36) Sasaki, K.-i.; Kato, K.; Tokura, Y.; Suzuki, S.; Sogawa, T. Phys. Rev. B 2012, 85, 075437.

(37) Vandescuren, M.; Hermet, P.; Meunier, V.; Henrard, L.; Lambin, P. Phys. Rev. B 2008, 78, 195401. 
(38) Gao, L.; Ren, W.; Xu, H.; Jin, L.; Wang, Z.; Ma, T.; Ma, L.-P.; Zhang, Z.; Fu, Q.; Peng, L.-M.; Bao, X.; Cheng, H.-M. Nat Commun 2012, 3, 699.

(39) Wang, Y.; Zheng, Y.; Xu, X.; Dubuisson, E.; Bao, Q.; Lu, J.; Loh, K. P. ACS Nano 2011, 5, 9927-9933, PMID: 22034835.

(40) Rakic, I. S.; Capeta, D.; Plodinec, M.; Kralj, M. Carbon 2016, 96, 243 - 249.

(41) Blake, P.; Hill, E. W.; Castro Neto, A. H.; Novoselov, K. S.; Jiang, D.; Yang, R.; Booth, T. J.; Geim, A. K. Applied Physics Letters 2007, 91, 6.

(42) Yoon, D.; Moon, H.; Son, Y.-W.; Choi, J. S.; Park, B. H.; Cha, Y. H.; Kim, Y. D.; Cheong, H. Phys. Rev. B 2009, 80, 125422.

(43) Venezuela, P.; Lazzeri, M.; Mauri, F. Phys. Rev. B 2011, 84, 035433.

(44) Cancado, L. G.; Takai, K.; Enoki, T.; Endo, M.; Kim, Y.; Mizusaki, H.; Jorio, A.; Coelho, L. N.; Magalhaes-Paniago, R.; Pimenta, M. A. Applied Physics Letters 2006, 88, 163106.

(45) Liu, C.; Ma, Y.; Li, W.; Dai, L. Applied Physics Letters 2013, 103, 213103.

(46) Wang, Y. Y.; Ni, Z. H.; Shen, Z. X.; Wang, H. M.; Wu, Y. H. Applied Physics Letters 2008, $92,043121$.

(47) Das, A.; Chakraborty, B.; Sood, A. K. Bulletin of Materials Science 2008, 31, 579-584.

(48) Rossi, E.; Das Sarma, S. Phys. Rev. Lett. 2008, 101, 166803.

(49) Burson, K. M.; Cullen, W. G.; Adam, S.; Dean, C. R.; Watanabe, K.; Taniguchi, T.; Kim, P.; Fuhrer, M. S. Nano Letters 2013, 13, 3576-3580, PMID: 23879288.

(50) Thomsen, C.; Reich, S. In Light Scattering in Solids IX: edited by M. Cardona; SpringerVerlag: Berlin, 2007; Vol. 108, pp 115-235. 
(51) Mak, K. F.; Shan, J.; Heinz, T. F. Phys. Rev. Lett. 2011, 106, 046401.

(52) Dutta, S.; Lakshmi, S.; Pati, S. K. Physical Review B 2008, 77, 073412.

(53) Alfonsi, J.; Meneghetti, M. New Journal of Physics 2012, 14, 053047.

(54) Piao, Y.; Meany, B.; Powell, L. R.; Valley, N.; Kwon, H.; Schatz, G. C.; Wang, Y. Nat Chem 2013, 5, 840-845.

(55) Ghosh, S.; Bachilo, S. M.; Simonette, R. A.; Beckingham, K. M.; Weisman, R. B. Science 2010, 330, 1656-1659.

(56) Imamura, G.; Saiki, K. ACS Applied Materials \& Interfaces 2015, 7, 2439-2443, PMID: 25569142.

(57) Vinogradov, N. A.; Schulte, K.; Ng, M. L.; Mikkelsen, A.; Lundgren, E.; Martensson, N.; Preobrajenski, A. B. The Journal of Physical Chemistry C 2011, 115, 9568-9577.

(58) Larciprete, R.; Fabris, S.; Sun, T.; Lacovig, P.; Baraldi, A.; Lizzit, S. Journal of the American Chemical Society 2011, 133, 17315-17321, PMID: 21846143.

(59) Mühl, T.; Myhra, S. Nanotechnology 2007, 18, 155304.

(60) Nikitin, A.; Ogasawara, H.; Mann, D.; Denecke, R.; Zhang, Z.; Dai, H.; Cho, K.; Nilsson, A. Phys. Rev. Lett. 2005, 95, 225507.

(61) Elias, D. C.; Nair, R. R.; Mohiuddin, T. M. G.; Morozov, S. V.; Blake, P.; Halsall, M. P.; Ferrari, A. C.; Boukhvalov, D. W.; Katsnelson, M. I.; Geim, A. K.; Novoselov, K. S. Science 2009, 323, 610-613.

(62) Balog, R. et al. Nat Mater 2010, 9, 315-319.

(63) Haberer, D.; Vyalikh, D. V.; Taioli, S.; Dora, B.; Farjam, M.; Fink, J.; Marchenko, D.; Pichler, T.; Ziegler, K.; Simonucci, S.; Dresselhaus, M. S.; Knupfer, M.; Büchner, B.; Grüneis, A. Nano Letters 2010, 10, 3360-3366, PMID: 20695447. 
(64) Haberer, D. et al. Advanced Materials 2011, 23, 4497.

(65) Talirz, L. et al. ACS Nano 2017, 11, 1380-1388, PMID: 28129507.

(66) Horcas, I.; Fernandez, R.; Gomez-Rodriguez, J.; Colchero, J.; Gomez-Herrero, J.; Baro, A. Review of Scientific Instruments 2007, 78, 013705.

(67) Her, M.; Beams, R.; Novotny, L. Physics Letters A 2013, 377, 1455 - 1458. 